\title{
ACEITAÇÕO DE QUIMIOTERAPIA POR BRASILEIRAS COM CÂNCER DE MAMA
}

Carolina Games Bensi, Arinilda Silva Campos, Renata Midori Harada, Karina Ragazzo Oliani, Rodrigo Perez Ranzatti, Eliana Sueco Tibana Samano, Marina Sahade Gonçalves, Auro del Giglio*

Trabalho realizado no Instituto de Oncologia da Faculdade de Medicina do ABC

* Correspondência: Av. Rebouças, 3387

Cep 05410-040, São Paulo, SP

\section{RESUMO}

INTRODuçäo. $O$ câncer de mama é 0 segundo tipo de câncer mais incidente e a primeira causa de morte por câncer entre as mulheres brasileiras. Na perspectiva do médico, a indicação da quimioterapia adjuvante como parte de seu tratamento deve se pautar pelos seus riscos e benefícios. Do ponto de vista da paciente, entretanto, em nosso meio, há poucos dados acerca de suas opiniões quanto à magnitude dos benefícios e riscos associados a este tratamento.

Objetivos. Avaliar o benefício mínimo necessário para que pacientes com câncer de mama que já tenham realizado quimioterapia aceitem-na novamente, caso hipoteticamente isso se fizesse necessário e conhecer fatores que, eventualmente, poderiam influenciar nessa decisão.

Métodos. Foram aplicados questionários a 52 pacientes sobre dados gerais e clínicos de cada paciente, além de perguntas a respeito do benefício mínimo necessário para a aceitação de uma eventual nova quimioterapia.

REsultados. 75\% fariam uma nova quimioterapia mesmo se ela não alterasse a chance de recidiva da doença em cinco anos; metade das pacientes a fariam mesmo que esta não aumentasse a sua sobrevida e $61,54 \%$ se submeteriam novamente a um tratamento quimioterápico mesmo que este não aumentasse a sua chance de cura. A aceitação de uma nova quimioterapia que produzisse mínimos benefícios foi significativamente maior dentre pacientes mais idosas, com menor escolaridade e entre aquelas que não receberam quimioterapia prévia com adriamicina.

Conclusão. Mesmo por um benefício muito pequeno, a grande maioria das pacientes estudadas aceitaria ser submetida a uma nova quimioterapia, se indicada por seus médicos.

UNITERMOS: Câncer da mama. Quimioterapia. Satisfação do paciente.

\section{INTRODUÇÃO}

No Brasil, analisando-se as taxas de mortalidade das macro-regiões, o câncer está sempre entre as principais causas de morte', constituindo atualmente a segunda causa de morte por doença no país ( $12,7 \%$ em 2000)', sendo superado apenas pelas doenças cardiovasculares'. Segundo o Instituto Nacional do Câncer, a estimativa para o ano de 2003 é o surgimento de 402.190 novos casos de câncer, sendo que destes provavelmente I 26.960 evoluirão a óbito'. Entre as mulheres, o câncer de mama é o segundo mais incidente e a primeira causa de morte por câncer'.

A quimioterapia adjuvante nos casos de câncer de mama traz benefícios que variam de acordo com o risco prévio da paciente, a histologia do tumor e a presença ou não de receptores para estrógeno e progesterona ${ }^{2}$. Embora a metade dos casos de câncer de mama seja diagnosticado em estadio avançado', nas mulheres com menos de 50 anos em que o diagnóstico é feito em estadio inicial, a quimioterapia adjuvante aumenta em $10 \%$ a sobrevida em dez anos ${ }^{2}$ e em 15,2\% em cinco anos ${ }^{3}$. Já para o grupo de 50 a 69 anos, aumenta em 3\% a sobrevida em dez anos, o que corresponde a um ganho real de I a 3 anos na expectativa de vida ${ }^{2}$. No entanto, essa mesma terapia apresenta alguns efeitos adversos agudos, como alopécia, náuseas, adinamia e maior risco de infecção, além de outros a longo prazo, como disfunção cardíaca e mielodisplasia ${ }^{2,3,4}$. Embora estes efeitos colaterais sejam freqüentemente bem controlados por medicações administradas em conjunto com as sessões de quimioterapia, se não forem corretamente abordados podem acarretar uma piora importante da qualidade de vida destas mulheres.

Portanto, ao se prescrever a quimioterapia adjuvante a pacientes com câncer de mama, é preciso ponderar seus benefícios e malefícios, levando-se sempre em consideração as opiniões e as vontades das pacientes. Essa atitude torna-se ainda mais importante quando se trata de uma segunda quimioterapia pela qual a paciente passa, como por exemplo, em casos de recidivas, já que o valor mais ou menos traumatizante atribuído à experiência varia de acordo com a vivência e crenças pessoais de cada paciente, influenciando muito na decisão de aceitar ou não esse segundo tratamento. Entretanto, há poucos estudos na literatura internacional que avaliam a opinião da paciente com câncer a respeito desta situação e qual o benefício mínimo necessário para que ela aceite uma nova quimioterapia.

Jansen et al. ${ }^{3}$ verificaram que mulheres previamente submetidas à quimioterapia aceitariam iniciar um novo tratamento quimioterápico com um mínimo benefício de 1\%. Trinta e nove por cento destas pacientes aceitariam mesmo sem nenhum benefício.

No entanto, em um estudo de Duric e Stockler ${ }^{2}$, apenas 1\% a 2\% das mulheres previamente submetidas à quimioterapia aceitariam fazer um novo ciclo de tratamento sem nenhum benefício, enquanto que 
BENSI CG ET AL.

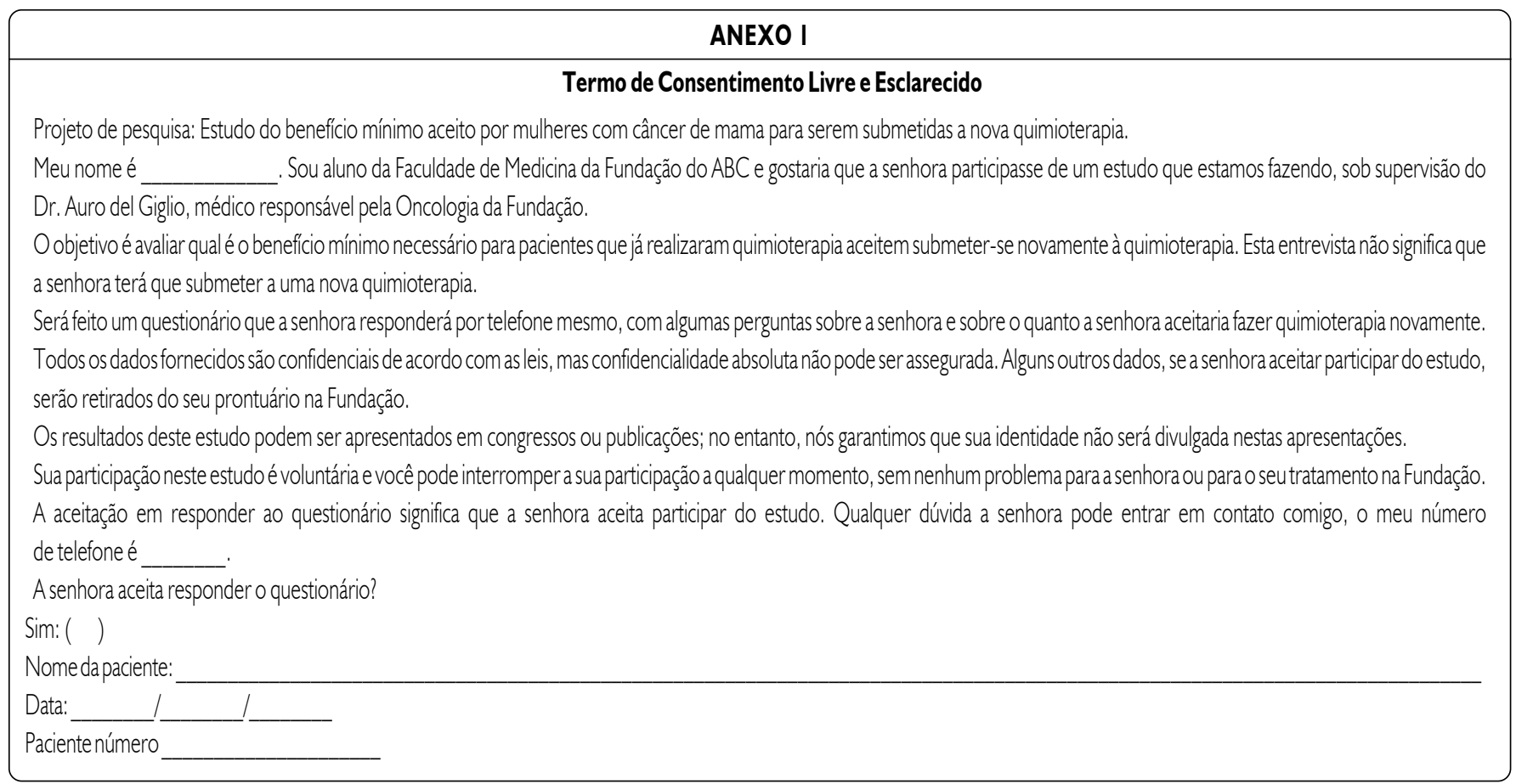

metade das mulheres considera o aumento de $1 \%$ na sobrevida ou cura ou um prolongamento de 6 meses em sua expectativa de vida suficientes para aceitar realizar o procedimento quimioterápico².

Outro estudo $0^{6}$ verificou que as mulheres aceitariam fazer quimioterapia se esta Ihes proporcionasse um aumento na expectativa de vida de 3 a 6 meses e uma redução do risco de recorrência de $0,5 \%$ a 1,0\%; entretanto, no grupo estudado, houve uma grande variação na taxa de aceitação, uma vez que $27 \%$ das mulheres não aceitariam menos que um ano de aumento na expectativa de vida ou menos que $5 \%$ de redução no risco de recorrência.

Assim, de acordo com a literatura internacional, grande parte das mulheres previamente submetidas à quimioterapia parece aceitar um novo ciclo de tratamento com mínimos benefícios. Na literatura nacional, porém, nenhuma referência a tal assunto foi encontrada.

Isso motivou o desenvolvimento desta pesquisa, pois consideramos de extrema importância o conhecimento do perfil das pacientes do nosso meio. Portanto, o objetivo deste estudo foi avaliar - benefício mínimo necessário (chance de cura, aumento na sobrevida e diminuição na taxa de recidiva da doença) para que as pacientes com câncer de mama que já haviam realizado tratamento quimioterápico no nosso serviço aceitassem uma nova quimioterapia. Também se objetivou conhecer fatores que eventualmente poderiam influenciar nessa decisão.

\section{Métodos}

Foram selecionadas todas as pacientes com câncer de mama que haviam realizado tratamento quimioterápico no setor de Quimio- terapia do Instituto de Oncologia da Faculdade de Medicina do ABC, no período de junho de 2000 a junho de 2003, tendo sido seus nomes e telefones obtidos através do livro de registros de pacientes atendidas no setor. Totalizaram-se 268 pacientes. Neste processo, foram incluídas todas as pacientes com diagnóstico de câncer de mama, independente da idade, estadio e tipo histológico, submetidas à quimioterapia e que já haviam encerrado pelo menos o primeiro ciclo. Foram excluídas as pacientes que, segundo o relatório, não tinham capacidade mental ou física para responder o questionário, assim como aquelas que não haviam completado o primeiro ciclo da quimioterapia.

Realizamos um contato telefônico e, após a devida identificação do entrevistador e explicação do objetivo do estudo, de acordo com o modelo que segue em anexo (Anexo I), considerou-se a aceitação em participar da entrevista como um Termo de Consentimento Livre e Esclarecido (Anexo I).

Em seguida, foi aplicado um questionário de forma padronizada (Anexo 2) que consistia em duas partes, a primeira com dados gerais da paciente (idade, escolaridade, estado civil, raça e outros) e dados clínicos (estadio, número de ciclos, tipo de cirurgia realizada, entre outros que foram obtidos dos registros do prontuário). É importante ressaltar que, quanto ao tipo de quimioterapia recebida, as pacientes foram divididas em dois grupos: aquelas que receberam adriamicina e as que não receberam (as que receberam mais de um tipo de quimioterapia, sendo que um dos esquemas incluía a adriamicina, foram incluídas no primeiro grupo); o motivo de tal divisão foi o maior número de efeitos colaterais que a adriamicina acarreta, entre eles a queda de cabelos, a qual muitas vezes traz um maior desconforto à paciente 7 . 


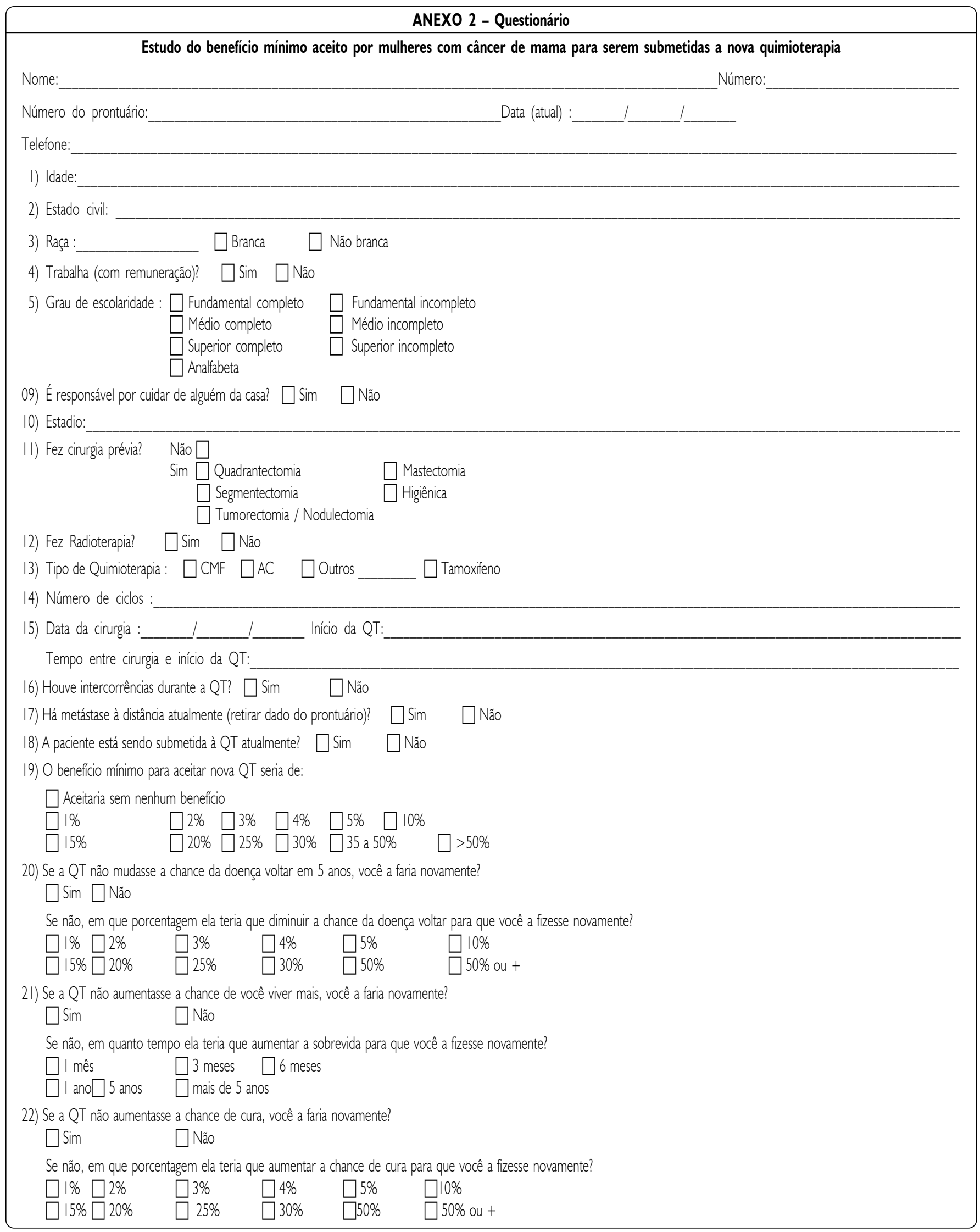


BeNSI CG ET AL.

A segunda parte continha questões que avaliavam o quanto de benefício seria necessário para que a paciente aceitasse receber um novo procedimento quimioterápico. Convém observar que benefício, em nosso estudo, foi definido como chance de cura, aumento na sobrevida ou diminuição na taxa de recidiva em cinco anos; desse modo, constavam perguntas sobre o benefício mínimo necessário e sobre o quanto de chance de cura ou de recidiva a quimioterapia teria que aumentar ou diminuir, respectivamente, para que ela se submetesse a um novo procedimento.

Todos os entrevistadores foram homogêneos na forma de questionar a paciente. Foi ainda explicado à participante que a entrevista não significava que ela teria que se submeter a uma nova quimioterapia, evitando-se que se criassem ansiedades desnecessárias.

As análises estatísticas pertinentes foram realizadas no programa NCSS 2000 - PASS 2000 (Utah, EUA). O estudo estatístico entre as variáveis discretas e contínuas foi feito através da análise de variância (ANOVA) e o estudo entre as variáveis discretas entre si através de teste de qui-quadrado. Consideramos como significativos valores de p inferiores a 0,05.

\section{Resultados}

Das 268 pacientes selecionadas, 160 foram excluídas por não terem sido localizadas, ou por problemas com o número de telefone ou por não atenderem às ligações ou por não residirem mais no mesmo local. Das 108 remanescentes, 46 haviam falecido e 10 não quiseram participar do estudo, tendo sido efetivamente entrevistadas 52.

\section{Análise das características demográficas}

Entre as pacientes entrevistadas, a média de idade encontrada foi de 55,65 \pm |I,8I anos, variando de 27 a 78 anos. A maioria era branca $(63,46 \%)$, morava com companheiro $(61,53 \%)$ e não trabalhava com remuneração $(92,30 \%)$. Trinta e duas pacientes $(61,53 \%)$ eram responsáveis por cuidar de alguém da casa. A maior parte tinha escolaridade inferior ao Ensino Fundamental completo $(65,38 \%)$. Estes e os demais dados demográficos estão descritos nas Tabelas | e 2. Com relação às características da quimioterapia recebida e o uso de hormonioterapia, os resultados seguem na Tabela 3.

Quanto às perguntas sobre o benefício mínimo necessário para aceitação de novo procedimento quimioterápico, verificamos que a maioria das pacientes $(38,46 \%$ ) aceitaria uma nova quimioterapia se esta trouxesse um benefício mínimo de $1 \%$. Nove pacientes $(17,3 \%)$ fariam uma nova quimioterapia mesmo sem nenhum benefício e uma paciente não faria quimioterapia novamente sob nenhuma circunstância (Tabela 4).

Quando perguntamos se a paciente faria quimioterapia novamente mesmo que esta não mudasse a taxa recidiva da doença em cinco anos, a maioria das pacientes (75\%) aceitaria fazê-la. (Tabela 5). Metade das pacientes faria uma nova quimioterapia mesmo que esta não aumentasse a sua sobrevida, enquanto 14 pacientes $(26,92 \%)$ necessitariam de um aumento na sobrevida de mais de cinco anos para aceitarem uma nova quimioterapia (Tabela 6).

\begin{tabular}{lc}
\hline \multicolumn{2}{c}{ Tabela I - Características demográficas: aspectos gerais } \\
\hline \multicolumn{1}{c}{ Características } & $\mathbf{N}(\%)$ \\
\cline { 1 - 2 } Média de idade & $55,65 \pm 11,81$ anos \\
Estado Civil & $32(61,54 \%)$ \\
Com companheiro & $20(38,46 \%)$ \\
Sem companheiro & \\
Raça & $33(63,46 \%)$ \\
Branca & $19(36,54 \%)$ \\
Não branca & \\
Trabalho & $4(7,69 \%)$ \\
Comremuneração & $48(92,31 \%)$ \\
Sem remuneração & \\
Escolaridade & $34(65,38 \%)$ \\
Atéfundamental completo & $9(17,31 \%)$ \\
Até médio completo & $6(11,54 \%)$ \\
Atésuperior completo & $3(5,77 \%)$ \\
Analfabeto & \\
Cuida de alguém da casa? & $32(61,54 \%)$ \\
Sim & $20(38,46 \%)$ \\
Não &
\end{tabular}

Legenda: N=número

Tabela 2 - Dados demográficos: dados clínicos

\begin{tabular}{lc}
\hline \multicolumn{2}{c}{ Tabela 2 - Dados demográficos: dados clínicos } \\
\hline Dados clínicos & $\mathbf{N}(\%)$ \\
Estadiamento & $1(1,92 \%)$ \\
\hline II & $17(32,69 \%)$ \\
IV & $8(15,39 \%)$ \\
Sem dados & $15(28,85 \%)$ \\
Metástase & $11(21,15 \%)$ \\
Sim & \\
Não & $15(28,85 \%)$ \\
Sem dados & $33(63,46 \%)$ \\
Cirurgia prévia & $4(7,69 \%)$ \\
Não fez & \\
Segmentectomia & $5(9,62 \%)$ \\
Quadrantectomia & $3(5,77 \%)$ \\
Mastectomía & $13(25 \%)$ \\
Mastectomiahigiênica & $27(51,92 \%)$ \\
Sem dados & $1(1,92 \%)$ \\
Há quanto tempo (média) & $3(5,77 \%)$ \\
Radioterapia prévia & 44,93 meses $( \pm 27,50)$ \\
Sim & \\
Não & $49(94,23 \%)$ \\
Sem dados & $2(3,85 \%)$ \\
\hline Legenda: N=número & $1(1,92 \%)$ \\
\hline
\end{tabular}

Trinta e duas pacientes $(61,54 \%)$ se submeteriam a uma nova quimioterapia mesmo que esta não aumentasse a chance de cura da doença. As demais aceitariam se submeter novamente à quimioterapia se esta Ihes propiciasse alguma chance de cura (Tabela 7).

Correlações entre características demográficas e beneficio mínimo necessário para aceitar nova quimioterapia 


\begin{tabular}{|c|c|}
\hline Dados & $\mathbf{N}(\%)$ \\
\hline $\begin{array}{l}\text { Tipo de quimioterapia } \\
\text { Comadriamicina } \\
\text { Semadriamicina } \\
\text { Sem dados }\end{array}$ & $\begin{array}{l}29(55,77 \%) \\
19(36,54 \%) \\
4(7,69 \%)\end{array}$ \\
\hline $\begin{array}{l}\text { Número de esquemas realizados } \\
\text { I esquema } \\
\text { 2 esquemas } \\
\text { 3 esquemas } \\
\text { Sem dados }\end{array}$ & $\begin{array}{l}33(63,46 \%) \\
15(28,85 \%) \\
1(1,92 \%) \\
3(5,77 \%)\end{array}$ \\
\hline $\begin{array}{l}\text { Número de ciclos realizados } \\
\text { Menos que } 6 \text { ciclos } \\
6 \text { ciclos } \\
\text { Mais que } 6 \text { ciclos } \\
\text { Sem dados } \\
\text { Média de ciclos }\end{array}$ & $\begin{array}{c}3(5,77 \%) \\
27(51,92 \%) \\
17(32,70 \%) \\
5(9,61 \%) \\
7,1\end{array}$ \\
\hline $\begin{array}{l}\text { Fez quimioterapia há quanto tempo (média) } \\
\text { Intercorrências durante a quimioterapia? } \\
\text { Sim } \\
\text { Não }\end{array}$ & $\begin{array}{c}41,68 \text { meses } \pm 23,46 \\
34(65,38 \%) \\
18(34,62 \%)\end{array}$ \\
\hline $\begin{array}{l}\text { Em quimioterapia atualmente? } \\
\text { Sim } \\
\text { Não }\end{array}$ & $\begin{array}{c}4(7,69 \%) \\
48(92,31 \%)\end{array}$ \\
\hline $\begin{array}{l}\text { Uso de Tamoxifeno } \\
\text { Sim } \\
\text { Não } \\
\text { Sem dados }\end{array}$ & $\begin{array}{c}26(50 \%) \\
23(44,23 \%) \\
3(5,77 \%)\end{array}$ \\
\hline $\begin{array}{l}\text { Uso de Femara } \\
\text { Sim } \\
\text { Não }\end{array}$ & $\begin{array}{c}5(9,62 \%) \\
47(90,38 \%)\end{array}$ \\
\hline
\end{tabular}

Legenda: $N=$ número

\begin{tabular}{cc}
\hline \multicolumn{2}{c}{$\begin{array}{c}\text { Tabela } 4 \text { - Benefício mínimo global necessário para } \\
\text { aceitar uma nova quimioterapia }\end{array}$} \\
\hline Benefício mínimo global & $\frac{\mathbf{N}(\%)}{9(17,31 \%)}$ \\
\hline Nenhumbenefício & $20(38,46 \%)$ \\
$1 \%$ de benefício & $9(17,31 \%)$ \\
Entre $1 \%$ e $50 \%$ de benefício & $\mid 2(23,08 \%)$ \\
$50 \%$ ou mais de benefício & $\mid(1,92 \%)$ \\
Nãofaria quimioterapia novamente & $\mid(1,92 \%)$ \\
Não sabe &
\end{tabular}

Tabela 5 - Redução no índice de recidiva em cinco anos necessária para aceitar uma nova quimioterapia

\begin{tabular}{lc}
\hline Redução de recidiva em cinco anos & $\mathbf{N}(\%)$ \\
\cline { 2 - 2 } Nenhuma chance & $39(75 \%)$ \\
$1 \%$ de chance & $1(1,92 \%)$ \\
Entre l\% e $50 \%$ de chance & $4(7,70 \%)$ \\
$50 \%$ ou mais de chance & $7(13,46 \%)$ \\
Nãofaria quimioterapia novamente & $1(1,92 \%)$ \\
\hline Legenda: N=número &
\end{tabular}

Tabela 6-Aumento de sobrevida necessário para aceitar uma nova quimioterapia

\begin{tabular}{lc}
\hline \multicolumn{1}{c}{ Aumento de sobrevida } & $\mathbf{N}(\%)$ \\
\cline { 3 - 3 } Semaumento & $26(50 \%)$ \\
I mês a 5 anos & $9(17,31 \%)$ \\
Mais de 5 anos & $14(26,92 \%)$ \\
Nãofaria quimioterapia novamente & $1(1,92 \%)$ \\
Não sabiam & $2(3,85 \%)$ \\
\hline Legenda: N=número &
\end{tabular}

Tabela 7 - Chance de cura necessária para aceitar uma nova quimioterapia

\begin{tabular}{lc}
\multicolumn{1}{c}{ Chance de cura } & $\mathbf{N}(\%)$ \\
Nenhumachance & $32(61,54 \%)$ \\
$1 \%$ de chance & $5(9,62 \%)$ \\
$1 \%$ a $50 \%$ de chance & $7(13,46 \%)$ \\
$50 \%$ ou mais de chance & $6(11,54 \%)$ \\
Nãofaria quimioterapia novamente & $1(1,92 \%)$ \\
Não sabia & $\mid(1,92 \%)$ \\
\hline egenda: N=número &
\end{tabular}

Analisamos a relação entre o beneficio mínimo necessário para aceitar nova quimioterapia (redução do índice de recidiva, aumento da expectativa de vida e chance de cura) e as características demográficas. Não encontramos correlação com raça, estado civil, trabalho e ser responsável por cuidar de alguém da casa. No entanto, encontramos associação significativa entre aceitar nova quimioterapia, mesmo que esta não mude o índice de recidiva em cinco anos, e ter mais idade $(p=0,0 \mathrm{I})$. Constatamos ainda que pacientes com menor escolaridade tendem a aceitar nova quimioterapia mesmo por um menor tempo de sobrevida $(p=0,023)$. Ao analisarmos a correlação com os dados clínicos, tais como estadiamento, realização de radioterapia, tipo de cirurgia prévia e há quanto tempo esta havia sido realizada a última quimioterapia, não encontramos associações significativas.

\section{Correlações entre dados referentes à quimioterapia $\mathrm{e}$ hormonioterapia e beneficio mínimo necessário para aceitar nova quimioterapia}

Quando correlacionamos o tipo de quimioterapia administrada e o beneficio mínimo aceitável, verificamos que as pacientes que realizaram quimioterapia sem adriamicina aceitariam um novo tratamento, mesmo que $o$ índice de recidiva em cinco anos não se altere $(p=0,0 \mid 8)$. Não encontramos associação entre número de ciclos e esquemas, tempo transcorrido da realização da última quimioterapia, realização de quimioterapia no momento da entrevista e uso de tamoxifeno e femara.

\section{Discussão}

O modo como a paciente encara uma quimioterapia influencia futuramente não apenas em sua adesão maior ou menor ao tratamento, mas também em sua aceitação ou não, caso necessário, de um segundo procedimento quimioterápico ${ }^{3}$.

A grande maioria de nossas pacientes aceitaria passar por nova quimioterapia, se necessário fosse, por benefícios muito pequenos. A nosso ver, o benefício de uma quimioterapia materializa-se por um aumento da sobrevida, por uma diminuição na chance de recidiva 
BENSI CG ET AL.

(por nós denominado como "a chance da doença voltar") e pela chance de cura. De fato, cerca de $75 \%$ delas aceitariam um novo procedimento quimioterápico mesmo que este não mudasse a chance de sua doença recair. Jasen et al. relatam em sua experiência que 39\% das pacientes por eles estudadas aceitariam se submeter novamente à quimioterapia mesmo que esta não produzisse benefício em termos de sobrevida livre de doença em cinco anos ${ }^{3}$. A maioria das nossas pacientes $(61,54 \%)$ aceitaria uma nova quimioterapia mesmo que este tratamento não aumentasse sua chance de cura. Da mesma forma, o mínimo benefício necessário conforme visto subjetivamente pelas pacientes para que a maioria delas aceitasse se submeter a uma nova quimioterapia, foi de apenas $1 \%$, sendo este o mesmo valor citado, em média, por outros estudos $^{3,6}$. É possível que esta freqüente anuência de pacientes com câncer de mama já submetidas anteriormente à quimioterapia em se submeter novamente a um tratamento tão tóxico seja explicada pelo fato de que a quimioterapia parece oferecer uma maior sensação de controle sobre suas vidas, dando-Ihes uma posição ativa perante a doença e ajudando-as a lidar com o sentimento de impotência ${ }^{7}$.

Ao contrário do que relatam outros estudos $3,6,8,9$, observamos que as mulheres com mais idade aceitam uma nova quimioterapia mais facilmente, mesmo que esta não mude a chance de recidiva.

Também observamos que um menor grau de escolaridade se correlacionou com a aceitação de um menor aumento no tempo de sobrevida, o que não foi encontrado na literatura ${ }^{6,8,9}$. É possível que os trabalhos na literatura feitos em países desenvolvidos (EUA, Austrália e Holanda) não tivessem incluído pacientes de baixos níveis de escolaridade como é o caso da maioria de nossas pacientes. Desta forma, acreditamos ser este trabalho importante por incluir uma significante fração de nossa população, para a qual os dados de trabalhos presentes na literatura não são extrapoláveis.

Outra associação relevante foi a de que pacientes que haviam sido submetidas à quimioterapia com adriamicina precisariam de maiores benefícios do que as que não a receberam. Se considerarmos que este quimioterápico pode causar desconfortáveis efeitos colaterais, como alopécia e emese $e^{4}$, acreditamos que podemos explicar este achado. De fato, Coates e Simes relatam que mulheres submetidas a toxicidades menos intensas necessitam de benefícios menores em relação ao incremento de sobrevida que a quimioterapia Ihes propiciaria ${ }^{8}$.

Os fatores demográficos como ser branco ou não e estado civil, aparentemente, não influenciaram nas respostas das pacientes, o que condiz com estudos de Lindley et al. ${ }^{9}$ Quanto a ser responsável por cuidar de alguém, Coates e Simes ${ }^{8}$ referiram existir uma relação com o benefício mínimo necessário para receber uma nova quimioterapia, de modo que quando existe tal responsabilidade, as pacientes tenderiam a aceitar um menor benefício; no entanto, este achado não foi reproduzido entre as nossas pacientes. Adicionalmente, o fato de ter realizado ou não hormonioterapia também não demonstrou relevância, assim como citam novamente Coates e Simes ${ }^{8}$.

\section{Conclusões}

I. A maioria das pacientes por nós estudadas aceitaria um novo procedimento quimioterápico mesmo com poucos/nulos benefícios.

2. Para pacientes de maior idade, uma menor taxa de remissão da doença bastaria para que aceitassem uma nova quimioterapia.

Conflito de interesse: não há.

\section{SUMMARY \\ ACCEPTANCE OF CHEMOTHERAPY BY Brazilian WOMEN WITH BREAST CANCER}

INTRODUCTION. Breast cancer has the second highest incidence and is the first cause of death by cancer among Brazilian women. Under the physician's perspective chemotherapy should be indicated according to the risk benefit ratio in each case. However, from the Brazilian breast cancer patient's perspective this risk benefit ratio requires further classification.

OBJECTIVE. In patients with breast cancer who have already received chemotherapy evaluate the minimum benefit considered necessary for a renewed administration and learn which factors may influence this decision.

METHODS. we surveyed 53 patients comprising general, clinical and cancer related variables, as well as issues related to the minimal expected benefit which would induce the patient to agree to undergo chemotherapy.

RESULTS. 75\% would accept to receive chemotherapy again even if it would not change their probability of relapse, 50\% would accept chemotherapy again, even though it would not increase survival rates. Similarly, 81.54\% would submit once more to chemotherapy even though it would not increase their probability of cure. Acceptance of a new chemotherapy treatment yielding minimal benefits correlated significantly with more age, with a lower educational level and with no previous administration of Adriamycin.

CONCLUSION. Even with a minimal benefit, the majority of formerly treated breast cancer patients are willing to submit to another chemotherapy treatment. [Rev Assoc Med Bras 2006; 52(I): 17-22]

KEY WORDS: Breast cancer. Chemotherapy. Patient satisfaction.

\section{REFERÊNCIAS}

I. Ministério da Saúde: Instituto Nacional do Câncer. Estimativas da Incidência e Mortalidade por Câncer no Brasil 2003 [citado Nov 2003]. Disponível em: http://www.inca.gov.br/estimativas/2003.

2. Duric V, Stockler M. Patients' preferences for adjuvant chemotherapy in early breast cancer: a review of what makes it worthwhile. Lancet Oncol 2001;2( I I):691-7.

3. Jansen SJ, Kievit J, Nooij MA, De Haes JC, Overpelt IME, Van Slooten H, et al. Patients preferences for adjuvant chemotherapy in early breast cancer: is treatment worthwhile? Br J Cancer 200 I ;84( I 2): I 577-85.

4. Rivera E. Liposomal anthracyclines in metastatic breast cancer: clinical update. Oncologist 2003;8(2):3-9.

5. Stone P, Richardson A, Ream E, Smith AG, Kerr DJ, Kearney N. Cancerrelated fatigue: inevitable, unimportant and untreatable? Results of a multi-centre patient survey. Cancer fatigue forum. Ann Oncol 2000; I I (8): 97। -5.

6. Ravdin PM, Siminoff IA, Harvey JA. Survey of breast cancer patients concerning their knowledge and expectations of adjuvant therapy. J Clin Oncol 1998: 16(2):515-21.

7. Levine MN, Gafni A, Markham B, MacFarlane D. A bedside decision instrument to elicit a patient's preference concerning adjuvant chemotherapy for breast cancer. Ann Intern Med 1992; I 1 7(1):53-8.

8. Coates AS, Simes RJ. Patient preferences for adjuvant chemotherapyof early breast cancer: how much benefit is needed? J Natl Cancer Inst Monogr 2001; 30(I): 146-52.

9. Lindley C, Vasa S, Sawyer WT, Winer EP. Quality of life and preferences for treatment follow ng systemic adjuvant therapy for early breast cancer. J Clin Oncol 1998; I 6(10): I380-7.

Artigo recebido: 26/08/04

Aceito para publicação: 16/09/05 